\title{
Simulating Shock to Detonation Transition: Algorithm and Results
}

\author{
Juhua Zhang, ${ }^{*}, 1$ Zhuping Duan, ${ }^{*}$ and Jing Ding $\dagger$ \\ ${ }^{*}$ LNM, Institute of Mechanics, CAS, Beijing 100080, China; and †Beijing Institute \\ of Technology, Beijing 100081, China
}

Received February 24, 1998; revised November 23, 1998

\begin{abstract}
An algorithm based on flux-corrected transport and the Lagrangian finite element method is presented for solving the problem of shock dynamics. It is verified through the model problem of one-dimensional strain elastoplastic shock wave propagation that the algorithm leads to stable, non-oscillatory results. Shock initiation and detonation wave propagation is simulated using the algorithm, and some interesting results are obtained. (c) 1999 Academic Press
\end{abstract}

\section{INTRODUCTION}

This paper presents a numerical simulation for the shock to detonation transition and detonation wave propagation in high-energy solids. Shock dynamics of energetic materials has been studied a great deal in recent years (see [1-15]). There are two features that are different from the conventional dynamics of inert materials. First, the shock wave speed changes from the elastic shock wave speed to the detonation wave speed, typically from 3000 to $9000 \mathrm{~m} / \mathrm{s}$, and the shock pressure increases, typically from 1 to $40 \mathrm{GPa}$. Second, the source term governing the release of chemical energy must be present in the conservation equation. The presence of the source term in the governing balance equations presents new numerical problems which the theory has yet to address [16]. The Lagrangian finite element method (see $[3,17,18]$ ) has been successfully used for many years for hydrodynamics. A shock wave, as a mathematical discontinuity, cannot be directly accommodated in the continuum formulation that is the basis of many present-day wave codes. The straightforward application of this finite element method to the problems of shock dynamics will give rise to non-physical oscillations. To demonstrate this, consider, for example, onedimensional shock wave propagation in a $0.1-\mathrm{m}$-thick elastic plate. The theoretical shock

\footnotetext{
${ }^{1}$ To whom correspondence should be addressed at the Center of Excellence for Advanced Materials, Department of Applied Mechanics and Engineering Sciences, University of California, San Diego, La Jolla, CA 92093-0416.
} 

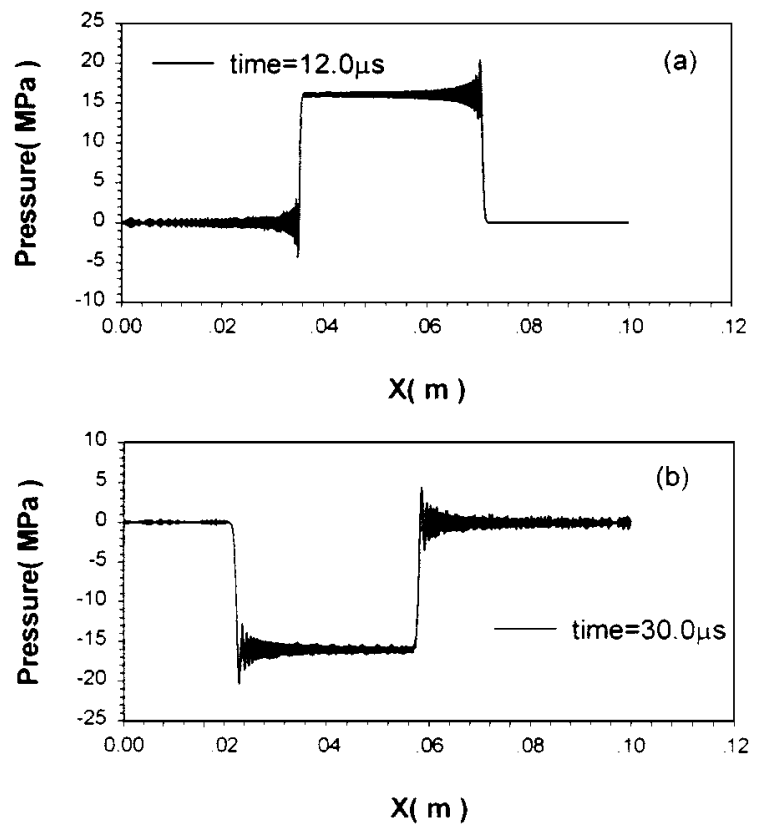

FIG. 1. Pressure profiles of 1-D strain elastic shock wave at time (a) $12.0 \mu$ s and (b) $30.0 \mu$ s without diffusion and anti-diffusion.

wave speed is $5927 \mathrm{~m} / \mathrm{s}$. A pressure of $1.6 \times 10^{7} \mathrm{~N} / \mathrm{m}^{2}$ is loaded on the front surface of the plate at 0 time for $6 \mu \mathrm{s}$. The wave profiles at $12.0 \mu \mathrm{s}$ (2000 times steps) and $30.0 \mu \mathrm{s}$ (5000 time steps) are shown, respectively, in Figs. 1a and 1b. The compressive wave has been changed into the tensile wave as shown in Fig. 1b because of free surface reflection. Figures $1 \mathrm{a}$ and $1 \mathrm{~b}$ reveal that there are two important features characteristic of the the finite element simulation. First, large amplitude oscillations appear everywhere in the domain. Second, the finite element solution has been identified with the correct wave speed even after the shock wave reflected from the free surface. To get around non-physical oscillations, an artificial bulk viscosity (see [3]) is usually introduced to smear the shock front over several mesh widths in calculations. The linear and quadratic terms of the artificial bulk viscosity are available. The former serves principally to spread the wave front over several elements in the direction of propagation and to lower the peak amplitude. The latter is used principally to suppress spurious oscillations behind the wave front. However, the artificial viscosity leads to excessive spreading of the shock profile, which will consequently introduce fictitious shock wave and detonation wave structures.

The flux-corrected transport (FCT) idea originates from Boris and Book [19, 20] and consists mainly of two stages, a transport-diffusive stage and an anti-diffusive stage. An equivalent but more descriptive interpretation of the FCT algorithm was given later by Zalesak [21]. Since then the algorithm has been enriched by many researchers; see, for example, McDonald [22], Giannakouros and Karniadakis [23], Salari and Steinberg [25], Odstrč̌l [24], and Löhner et al. [26, 27].

The finite element flux-corrected transport (FEM-FCT) algorithm with the Eulerian approach on unstructured grids [27] was well established by Löhner et al. [26] more than 10 years ago and has been extensively used since then. This method first expands the unknown at time level $n+1$ about the value at time level $n$ in a Taylor series, and then performs 
the spatial discretization via the Galerkin weighted residual method. The consistent-mass Taylor-Galerkin and lumped-mass Taylor-Galerkin are employed and combined in such a way that the high-order solution is used in smooth regions of the flow, whereas the low-order solution is favored near discontinuities. The two schemes are matched via the diffusion/antidiffusion steps. Since the lumped-mass Taylor-Galerkin cannot produce monotonic results for problems to be solved, the mass diffusion must be added to the lumped mass.

The Eulerian approach used in this method will result in the mass, momentum, and energy flow between elements.

The Lagrangian finite element method differs from the Eulerian method in the way in which elements are bound with deformation bodies, so there is no mass flow between elements. The Lagrangian element method has been extensively applied to impact problems (see $[3,8]$ ), since it does not need to redefine the geometry boundaries of bodies impacting together at a new time level. In this paper, we attempt to combine the FCT with the Lagrangian finite element discretization. Since the mass density flux and energy flux resulting from the mass flow between elements vanish in the Lagrangian approach, the FCT algorithm will be used to correct nodal momentums. Actually, only nodal velocities need to be corrected, because the nodal mass does not change with time.

This paper is organized as follows. The mathematical and numerical aspects of the finite element method are briefly treated in Section 2. The main steps incorporating FCT into the finite discretization are presented in Section 3, which ends with several elastoplastic shock wave results to verify the feasibility of the proposed algorithm. In Section 4, we describe the extra processes that are needed for simulating the shock to detonation transition. Finally, results and a brief discussion are presented in Section 5, followed by concluding remarks in Section 6.

\section{LAGRANGIAN FINITE ELEMENT DISCRETIZATION}

A material body $\Omega$ which occupies a finite region of Euclidean space is subjected to a prescribed body force $f^{k}$ and a surface loading $s^{k}$ which acts on $\phi_{1}$, where index $k$ ranging from 1 to 3 is the degree of freedom label. The problem is stated in terms of the principle of virtual work. The variational form of the momentum equation and the force boundary condition is

$$
\delta \pi=\int_{\Omega} \rho \ddot{x}^{k} \delta x_{k} d v+\int_{\Omega} \tau^{k m} \delta x_{k, m} d v-\int_{\Omega} \rho f^{k} \delta x_{k} d v-\int_{\phi_{1}} s^{k} \delta x_{k} d a,
$$

which vanishes at all points along the path of motion for all variations $\delta x_{k}$ satisfying the displacement boundary conditions on surface $\phi_{2}$. The integration is performed over the current configuration of the body $\Omega$ where $\rho$ is the mass density, $\ddot{x}$ is acceleration, $\tau^{k m}$ is Cauchy stress, and $f^{k}$ is the body force density in the current configuration. The sum convention is satisfied in the context.

\subsection{Spatial Discretization}

The Lagrangian dynamics finite element method is used to obtain an approximate solution. Examples of the application of the method are described in Refs. [3, 17]. The body $\Omega$ is divided into material subregions or elements with nodes at the vertices. Using interpolation or shape function within each element, a variable is defined over the element from 
the values it has at the nodes. Thus, the virtual displacement, velocity, and acceleration are given by

$$
\begin{aligned}
\delta x_{k}(t) & =\delta x_{k \alpha} \phi_{\alpha}, \\
\dot{x}^{k}(t) & =\dot{x}^{k \alpha} \phi^{\alpha}, \\
\ddot{x}^{k} & =\ddot{x}^{k \alpha} \phi^{\alpha},
\end{aligned}
$$

where $\phi^{\alpha}$ is the Galerkin shape function, $\alpha=1,2, \ldots, p$, in which $p$ accounts for the total number of the nodes. The values of $\phi^{\alpha}$ are the same for the different degrees of freedom of any node $\alpha$.

Substituting Eqs. (2), (3), (4) into Eq. (1) yields, for all virtual displacement satisfying the displacement boundary conditions on surface $\phi_{2}$, the approximate form of Eq. (1),

$$
\begin{aligned}
\delta \pi= & \int_{\Omega} \rho \ddot{x}^{k \beta} \phi^{\alpha} \phi^{\beta} \delta x_{k \alpha} d v+\int_{\Omega} \tau^{k m} \phi_{, m}^{\alpha} \delta x_{k \alpha} d v \\
& -\int_{\Omega} \rho f^{k} \phi^{\alpha} \delta x_{k \alpha} d v-\int_{\phi_{1}} s^{k} \phi_{, m}^{\alpha} \delta x_{k \alpha} d a_{m} \equiv 0 .
\end{aligned}
$$

This will result in

$$
\mathbf{M Q}=\mathbf{F}
$$

where

$$
\begin{gathered}
Q^{k \beta}=\ddot{x}^{k \beta} \\
M_{\alpha, \beta}^{k}=\int_{\Omega} \rho \phi^{\alpha} \phi^{\beta} \delta x_{k \alpha} d v \\
F^{k \alpha}=-\int_{\Omega} \tau^{k m} \phi_{, m}^{\alpha} d v+\int_{\Omega} \rho f^{k} \phi^{\alpha} d v+\int_{\phi_{1}} s^{k} \phi_{, m}^{\alpha} d a_{m}, \quad k=1,2,3 ; \alpha, \beta=1,2, \ldots, p .
\end{gathered}
$$

In Eq. (6), $\mathbf{M}$ is the nodal mass matrix, $\mathbf{F}$ is the nodal force vector, and $\mathbf{Q}$ is the nodal acceleration vector. If a diagonal-mass matrix is employed rather than the consistent-mass matrix implied in Eq. (6), we will obtain $3 \times p$ uncoupled Newtonian equations of motion for every degree of freedom of the nodes.

We are only concerned with the one-dimensional (1-D) case, including one-dimensional planar, cylindrical, and spherical symmetry in the following description.

\subsection{Mass Matrix}

The diagonal mass matrix is used in this work. The body $\Omega$ is divided into $N$ elements. Every node links two elements except the two end nodes that have only one element. The nodal mass is the summation of the the mass contributed by the element possessing the node. The two end nodes for one-dimensional plane symmetrical problems share the element mass equally. The element mass is weighted to its two end nodes in accordance with the distance between the node and the axis for one-dimensional cylindrical and spherical symmetry problem. If the two end node coordinates are $r_{1}$ and $r_{2}$, respectively, and the element mass 
is $q$, then the two end nodal masses supplied by the element possessing the nodes are, respectively,

$$
m_{1}=\frac{q}{4}+\frac{r_{1} q}{2\left(r_{1}+r_{2}\right)}, \quad m_{2}=\frac{q}{4}+\frac{r_{2} q}{2\left(r_{1}+r_{2}\right)}
$$

\subsection{Nodal Force}

It is convenient that the nodal physical coordinate $r_{1}$, and $r_{2}$ are transfered to the local coordinates -1 and 1 . The Lagrangian shape functions corresponding to two nodes are taken to have the linear form

$$
\phi_{1}=\frac{1}{2}(1-y), \quad \phi_{2}=\frac{1}{2}(1+y) .
$$

The physical coordinate for a given point within the element is given by

$$
r=\phi_{1} r_{1}+\phi_{2} r_{2}=\frac{1}{2}\left(r_{1}+r_{2}\right)+\frac{1}{2}\left(r_{2}-r_{1}\right) y \text {. }
$$

If $f^{k}=0$, then the nodal forces contributed by the element possessing the nodes are

$$
\left(\begin{array}{c}
F_{r_{1}} \\
F_{r_{2}}
\end{array}\right)=\int_{-1}^{1}\left(\begin{array}{cc}
\frac{d \phi_{1}}{d y} & \frac{\lambda}{r} \phi_{1} \\
\frac{d \phi_{2}}{d y} & \frac{\lambda}{r} \phi_{2}
\end{array}\right)\left(\begin{array}{c}
\sigma_{r} \\
\sigma_{\theta}
\end{array}\right) r^{\lambda} d y,
$$

where $\lambda=0,1,2$ corresponds, respectively, to one-dimensional planar, cylindrical, and spherical symmetry, and $\sigma_{r}$ and $\sigma_{\theta}$ are the stress components. For one-dimensional planar symmetry $\sigma_{\theta}=0$, we have

$$
\left(\begin{array}{c}
F_{r_{1}} \\
F_{r_{2}}
\end{array}\right)=\left(\begin{array}{c}
-\sigma_{r} \\
\sigma_{r}
\end{array}\right)
$$

The nodal force is the summation of the force contributed by the element possessing the nodal and external loading on the two end nodes. The nodal acceleration is

$$
a_{i}=\frac{F_{i}}{M_{i}} \quad(i=1,2, \ldots, p) .
$$

\subsection{Time Integration}

The central difference scheme is employed to get the nodal velocity and nodal displacement.

The nodal velocity is

$$
v_{i}\left(t+\frac{\Delta t}{2}\right)=v_{i}\left(t-\frac{\Delta t}{2}\right)+a_{i} \Delta t .
$$

The nodal displacement is

$$
u_{i}(t+\Delta t)=u_{i}(t)+v_{i}\left(t+\frac{\Delta t}{2}\right) \Delta t
$$


The time step $\Delta t$ must be taken to satisfy the Courant condition

$$
\Delta t=\zeta \frac{\min \Delta x}{\max (c, v)},
$$

where $\Delta x$ is the element length, $c$ is the characteristic wave speed of the material, $v$ is the nodal velocity, and $\zeta$ is taken to be 0.65 in this work.

\subsection{Strain Rate and Strain}

For one-dimensional planar symmetry, the strain rate of the element will be

$$
\dot{\epsilon}=\frac{d v}{d r}=\frac{d\left(v_{1} \phi_{1}+v_{2} \phi_{2}\right)}{d r}=\frac{v_{2}-v_{1}}{r_{2}-r_{1}} .
$$

The strain increment within one time step takes

$$
\Delta \epsilon=\dot{\epsilon} \Delta t
$$

\section{DIFFUSIVE AND ANTI-DIFFUSIVE ALGORITHM}

We consider here shock dynamics problems in inert and energetic materials. There are two approaches, the Boris and Book scheme [19, 20] and the Zalesak scheme [21], which can be adopted. Here we employ the approach of Boris and Book in formulating the diffusive and anti-diffusive algorithm.

\subsection{Main Steps}

In particular, the main steps of the diffusive and anti-diffusive Lagrangian finite element method are as follows:

(1) Input the characteristic constants of the material, initial condition, and boundary condition.

(2) Generate the finite element geometry including node label, element label, and node coordinate.

(3) Calculate the lumped mass at each of the nodes. The nodal masses are computed once at the start of the computations and remain fixed for all time.

(4) Compute the concentrated nodal force from the stresses in the element and the boundary loading.

(5) Compute the nodal acceleration through the nodal mass and force.

(6) Calculate the diffusive fluxes in accordance with the nodal velocity of the last time step, $f_{j}^{0}=m_{j+1} v_{j+1}^{0}-m_{j} v_{j}^{0}$, where $v_{j}$ is the nodal velocity of node $j$, and $m_{j}$ is the nodal mass of node $j$, which does not change with time.

(7) Integrate Eq. (15) to calculate the trial nodal velocity $v^{*}$.

(8) Through $v^{*}$, we get the anti-diffusive fluxes $f_{j}^{1}=m_{j+1} v_{j+1}^{*}-m_{j} v_{j}^{*}$.

(9) Apply diffusion, $\tilde{v}_{j}=v_{j}^{*}+\left(\eta / m_{j}\right)\left(f_{j}^{0}-f_{j-1}^{0}\right)$, where $\eta$ is the diffusive coefficient.

(10) Limit the anti-diffusive fluxes,

$$
f_{j}^{1} \rightarrow s \cdot \max \left\{0, \min \left[s \cdot \Delta_{j-1},\left|f_{j}^{1}\right|, s \cdot \Delta_{j+1}\right]\right\}
$$

where $s=\operatorname{sign}\left(\Delta_{j}\right), \Delta_{j}=m_{j+1} \tilde{v}_{j+1}-m_{j} \tilde{v}_{j}$. 
(11) Apply anti-diffusion to obtain the updated nodal velocity:

$$
v_{j}=\tilde{v}_{j}-\frac{\eta}{m_{j}}\left(f_{j}^{1}-f_{j-1}^{1}\right) .
$$

(12) Compute the nodal displacement from Eq. (17).

(13) Calculate the element strain rate and the element strain increment from Eqs. (19) and (20).

(14) The next time step length is generated from Eq. (18).

(15) Calculate the stresses or pressure within the element through the constitutive relation or the equation of state.

(16) If the calculation does not arrive at the final time, return to step (4) and then start a new time circle.

To conserve the linear momentum of the system, the suggestions proposed by one of this artical manuscript reviewers have included in the formulas using to calculate the diffusive and anti-diffusive fluxes.

\subsection{Model Problems}

To demonstrate the algorithm, we consider a problem of 1-D strain shock wave propagation within a half-infinite ideal elastoplastic medium. The initial conditions for the stress $\sigma$ and velocity $v$ are

$$
\sigma(t=0)=0 ; \quad v(t=0)=0
$$

The loading acting on the surface of the model body is given by

$$
p(x=0)=\left\{\begin{array}{ll}
1.6 \mathrm{GPa}, & \text { as } 0 \leq t \leq 6.0 \mu \mathrm{s} \\
0, & \text { as } t>6.0 \mu \mathrm{s}
\end{array} .\right.
$$

The constitutive model is given by

$$
\sigma=\left(K+\frac{4 G}{3}\right) \epsilon, \quad \text { as } \sigma<Y_{H},
$$

where $K$ is the volume modulus, $G$ is the shear modulus, $Y_{0}$ is the yield strength. $K, G$, and $Y_{0}$ are taken to be $166 \mathrm{GPa}, 81 \mathrm{GPa}$, and $800 \mathrm{MPa}$, respectively.

The Hugoniot elastic limit is

$$
Y_{H}=\frac{\left(K+\frac{4}{3} G\right) Y_{0}}{2 G}=1.35 \mathrm{GPa} .
$$

When the material density $\rho$ is taken to be $7800 \mathrm{~kg} / \mathrm{m}^{3}$, the elastic shock wave velocity $D_{e}=5927 \mathrm{~m} / \mathrm{s}$, and the plastic shock velocity $D_{p}=4613 \mathrm{~m} / \mathrm{s}$. The diffusive coefficient is taken to be 0.065 . The typical profiles of the pressure and velocity are shown in Figs. 2 and 3. Figures $2 \mathrm{a}$ and $3 \mathrm{a}$ display the twin-wave structure. The precursor elastic shock wave is followed by the plastic shock wave. The elastic unloading shock wave unloads the elastic wave and is shown in Figs. $2 b$ and $3 b$. Figures $2 c$ and $3 c$ exhibit that the internal impact of the elastic unloading wave against the elastic loading wave produces the weak elastic wave which propagates in the left direction, reducing the residual velocity of the medium. The calculation results are in good agreement with the theory's prediction. 

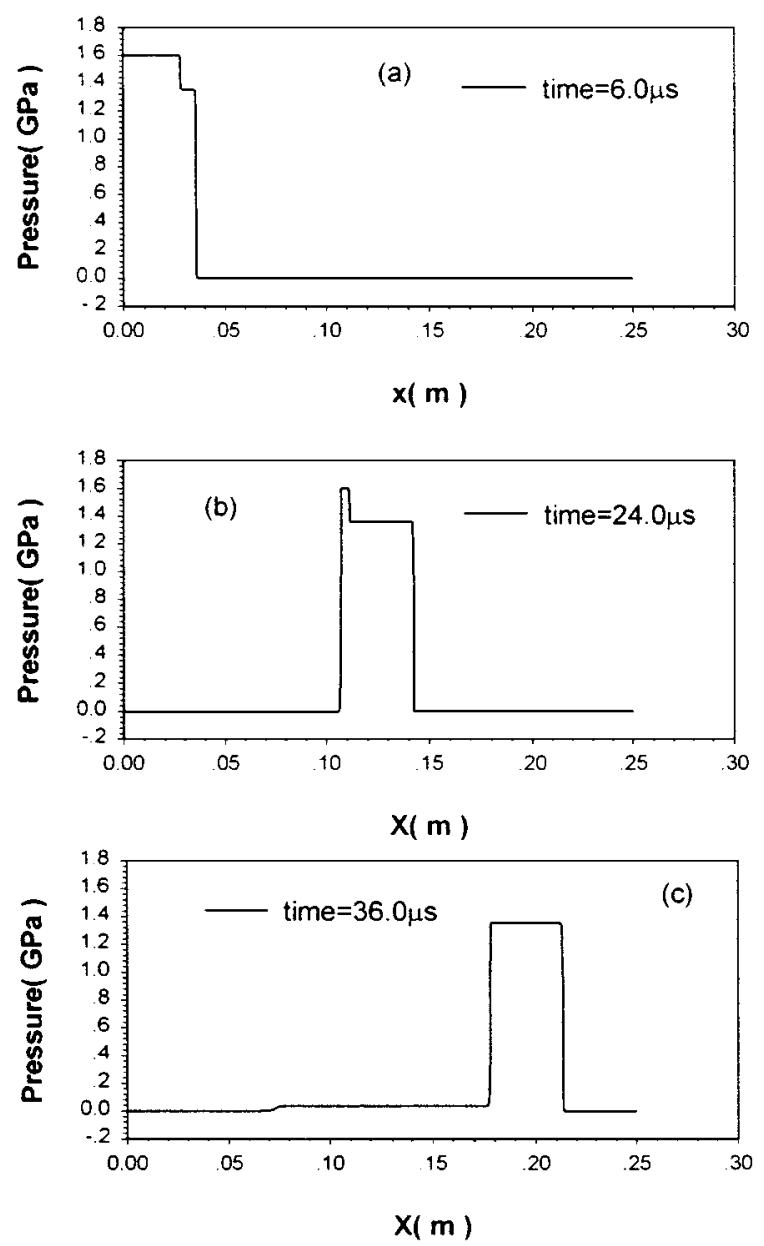

FIG. 2. Pressure profiles of 1-D strain elastoplastic shock wave at time (a) $6.0 \mu \mathrm{s}$, (b) $24.0 \mu \mathrm{s}$, and (c) $36.0 \mu \mathrm{s}$ via using diffusive and anti-diffusive algorithm.

\section{FORMULATION}

The Jones-Wilkins-Lee (JWL) form (see $[14,15]$ ) of equation of state is used to simulate the pressure-volume-energy relationship for the material in both the unreacted and reacted states. The equations of state for unreacted solid and reacted gas are given by Eqs. (25) and (26), respectively,

$$
\begin{aligned}
& P_{s}=A_{s}\left(1-\frac{W_{s}}{R_{1 s} V_{s}}\right) e^{-R_{1 s} V_{s}}+B_{s}\left(1-\frac{W_{s}}{R_{2 s} V_{s}}\right) e^{-R_{2 s} V_{s}}+\frac{W_{s} E_{s}}{V_{s}}, \\
& P_{g}=A_{g}\left(1-\frac{W_{g}}{R_{1 g} V_{g}}\right) e^{-R_{1 g} V_{g}}+B_{g}\left(1-\frac{W_{g}}{R_{2 g} V_{g}}\right) e^{-R_{2 g} V_{g}}+\frac{W_{g} E_{g}}{V_{g}},
\end{aligned}
$$

where $A_{s}, B_{s}, R_{1 s}, R_{2 s}, A_{g}, B_{g}, R_{1 g}, R_{2 g}, W_{s}, W_{g}$ are the material coefficients; $E_{s}, E_{g}$ are the internal energy per unit volume; and $V_{s}$ and $V_{g}$ are the specific volume,

$$
V_{s}=\frac{\rho_{0}}{\rho_{s}}=\frac{\rho_{0}}{\rho_{g}}=V_{g}=V
$$



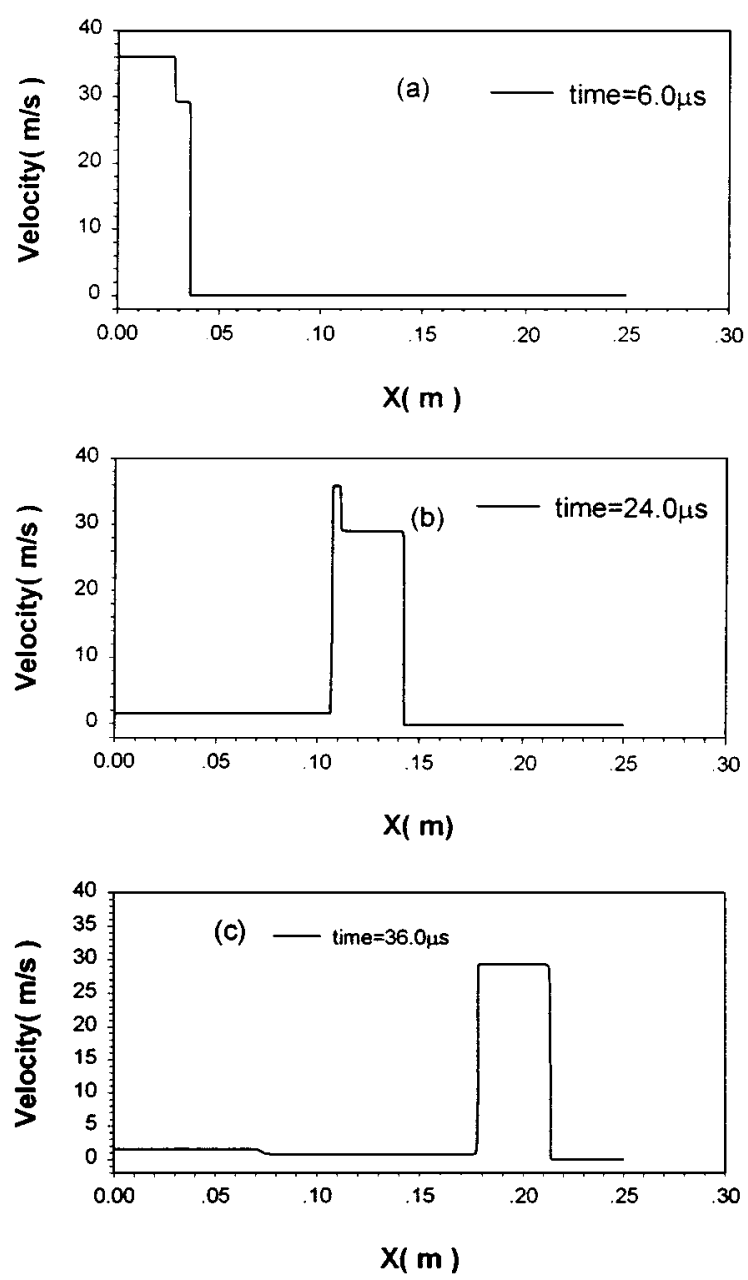

FIG. 3. Velocity profiles of 1-D strain elastoplastic shock wave at time (a) $6.0 \mu \mathrm{s}$, (b) $24.0 \mu \mathrm{s}$, and (c) $36.0 \mu \mathrm{s}$ via using diffusive and anti-diffusive algorithm.

where $\rho_{0}$ is the initial density of the material, and $\rho_{s}$ and $\rho_{g}$ are the current density of the material in unreacted solid and reacted gas, respectively. It is reasonable to recognize $\rho_{s}=\rho_{g}$ in the elements locating in the chemical reaction zone.

The chemical reaction is modeled by a reaction progress variable $F$, which denotes the mass fraction of the reacted material and takes a value between 0 (unreacted) and 1 (reacted completely). The ignition and growth model [14] controlling the transition from the unreacted phase to the reacted phase is used,

$$
\frac{d F}{d t}=I(1-F)^{2 / 9}\left(\frac{\rho}{\rho_{0}}-1\right)^{4}+G(1-F)^{2 / 9} F^{2 / 3} P^{z},
$$

where $I, G, Z$ are the reaction rate parameters. The reaction zone is treated as a mixture based on the two equations of state, so the state of the reaction zone is described by

$$
P=(1-F) P_{s}+F P_{g} .
$$


The third-order Adams-Bashforth scheme is used to update $F$ and $V$ on the new time level

$$
\begin{gathered}
F^{n+1}=F^{n}+\frac{\Delta t}{12}\left[5\left(\frac{d F}{d t}\right)^{n+1}+8\left(\frac{d F}{d t}\right)^{n}-\left(\frac{d F}{d t}\right)^{n-1}\right], \\
V^{n+1}-V^{n}=\frac{\Delta t}{12}\left(5 \dot{\epsilon}^{n+1}+8 \dot{\epsilon}^{n}-\dot{\epsilon}^{n-1}\right)
\end{gathered}
$$

where $\dot{\epsilon}$ is given by Eq. (19). If the heat conduction is neglected, the solid phase energy change in elements is given by

$$
d E_{s}=P_{s} d V_{s}
$$

which has the difference form

$$
E_{s}^{n+1}=E_{s}^{n}+\frac{1}{2}\left(P_{s}^{n+1}+P_{s}^{n}\right)\left(V^{n+1}-V^{n}\right) .
$$

Denoting the sum of the first and second term of Eq. (25) as $P_{1}$, we obtain

$$
P_{s}^{n+1}=P_{1}^{n+1}+\frac{W_{s} E_{s}^{n+1}}{V^{n+1}} .
$$

Substituting Eq. (34) into (33) yields

$$
E_{s}^{n+1}=\frac{E_{s}^{n}+0.5\left(P_{1}^{n+1}+P_{s}^{n}\right)\left(V^{n+1}-V^{n}\right)}{1-0.5 W_{s}\left(V^{n+1}-V^{n}\right) / V^{n+1}} .
$$

Substituting Eq. (35) into Eq. (34), we obtain the unreacted solid pressure $P_{S}$ on the new time level immediately. The reacted gas pressure $P_{g}$ is also obtained by the same steps. The acoustic speed is given by

$$
C^{2}=\frac{\partial P}{\partial \rho}=(1-F) \frac{\partial P_{s}}{\partial \rho}+F \frac{\partial P_{g}}{\partial \rho} .
$$

\section{RESULTS AND DISCUSSION}

The shock to detonation transition in an one-dimensional planar symmetry explosive charge is investigated. The initial conditions for the pressure $P$ and velocity $v$ are

$$
P(t=0)=0 ; \quad v(t=0)=0 .
$$

Corresponding to a 1-mm-thick copper flyer impacting the charge with the velocities $1.0 \times 10^{3}, 2.1 \times 10^{3}, 2.7 \times 10^{3}$, and $2.9 \times 10^{3} \mathrm{~m} / \mathrm{s}$, respectively, the loads acting on the surface of the model body are given by

$$
\begin{aligned}
& \text { (1) } p(x=0)= \begin{cases}6.29 \mathrm{GPa}, & \text { as } 0 \leq t \leq 0.57 \mu \mathrm{s} \\
0, & \text { as } t>0.57 \mu \mathrm{s}\end{cases} \\
& \text { (2) } p(x=0)= \begin{cases}15.7 \mathrm{GPa}, & \text { as } 0 \leq t \leq 0.57 \mu \mathrm{s} \\
0, & \text { as } t>0.57 \mu \mathrm{s}\end{cases}
\end{aligned}
$$


TABLE 1

The Parameters Used in the Equation of State and the Reaction Model [15]

\begin{tabular}{cccccc}
\hline$A_{s}(100 \mathrm{GPa})$ & $B_{s}(100 \mathrm{GPa})$ & $R_{1 s}$ & $R_{2 s}$ & $W_{s}$ & $A_{g}(100 \mathrm{GPa})$ \\
778.1 & -0.0503 & 11.3 & 1.13 & 0.8938 & 5.242 \\
$R_{2 g}$ & $W_{g}$ & $E_{s 0}\left(100 \mathrm{GPa} / \mathrm{m}^{3}\right)$ & $E_{g 0}\left(100 \mathrm{GPa} / \mathrm{m}^{3}\right)$ & $\rho_{0}\left(\mathrm{~kg} / \mathrm{m}^{3}\right)$ & $I\left(\mathrm{~s}^{-1}\right)$ \\
1.1 & 0.34 & $6.118 \times 10^{-3}$ & 0.085 & $1.712 \times 10^{3}$ & $44.6 \times 10^{6}$ \\
$B_{g}(100 \mathrm{GPa})$ & $R_{1 g}$ & $G\left(\mathrm{Mbar}^{-z} \mathrm{~s}^{-1}\right)$ & $Z$ & & \\
0.07678 & 4.2 & $414.0 \times 10^{6}$ & 2.0 & & \\
\hline
\end{tabular}

$$
\begin{aligned}
& \text { (3) } p(x=0)= \begin{cases}20.0 \mathrm{GPa}, & \text { as } 0 \leq t \leq 0.57 \mu \mathrm{s} \\
0, & \text { as } t>0.57 \mu \mathrm{s}\end{cases} \\
& \text { (4) } p(x=0)= \begin{cases}26.0 \mathrm{GPa}, & \text { as } 0 \leq t \leq 0.57 \mu \mathrm{s} \\
0, & \text { as } t>0.57 \mu \mathrm{s}\end{cases}
\end{aligned}
$$

The material parameters are given in Table 1 [15]. The diffusive coefficient is taken to be 0.125 . The profiles of pressure corresponding to the different initial impact pressure are shown in Figs. 4a to 4d. There are eight curves in each figure. The time corresponding to the first curves in Figs. $4 \mathrm{a}$ to $4 \mathrm{~d}$ is $0.6 \mu \mathrm{s}$. The time interval between two neighboring curves is $1.2 \mu \mathrm{s}$. The validity of the calculation is demonstrated by the fact that the four
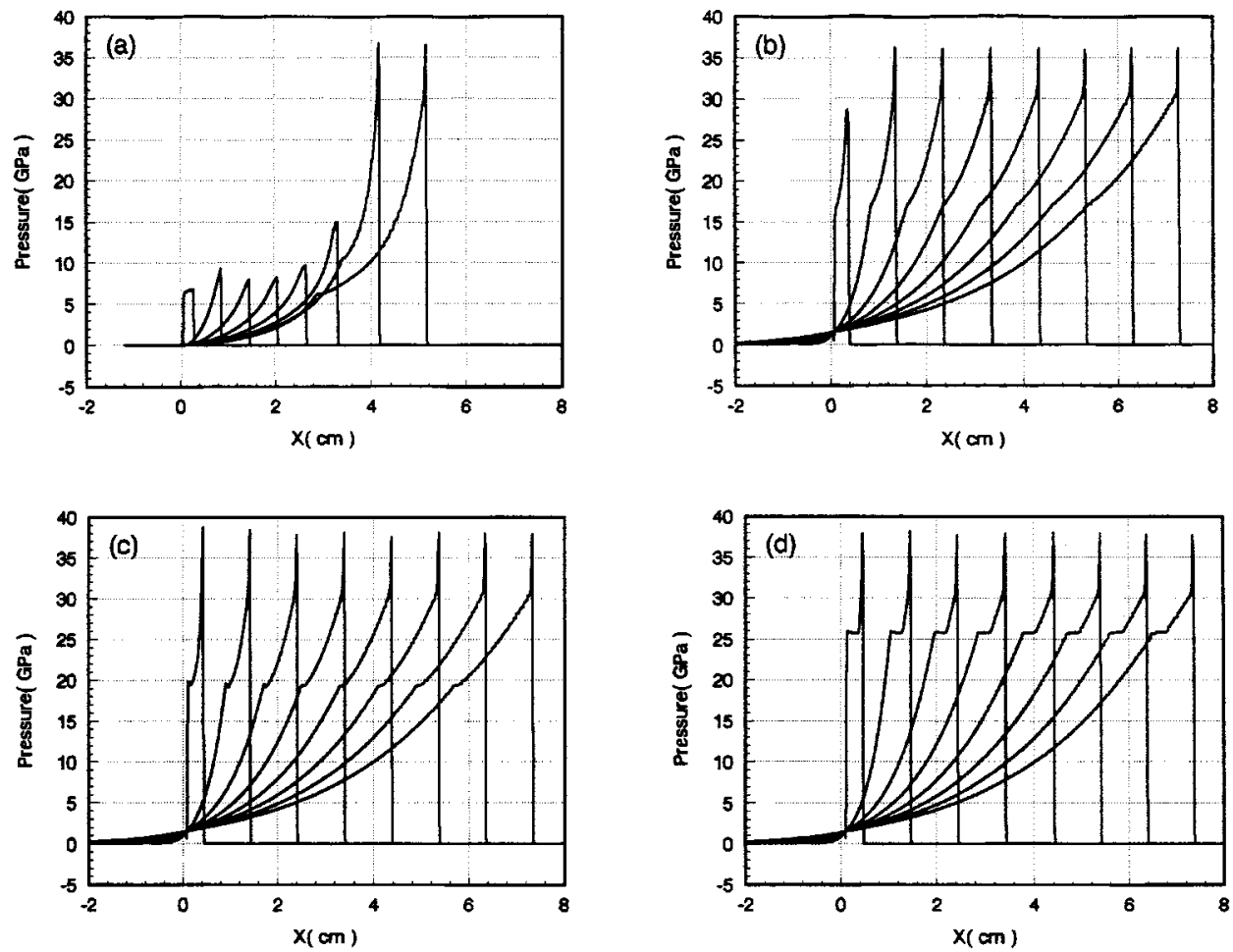

FIG. 4. Shock to detonation transition: pressure profiles. The sustained time of the loading is $0.57 \mu$ s. The loading pressure is (a) $6.29 \mathrm{GPa}$, (b) $15.7 \mathrm{GPa}$, (c) $20.0 \mathrm{GPa}$, and (d) $26.0 \mathrm{GPa}$. 


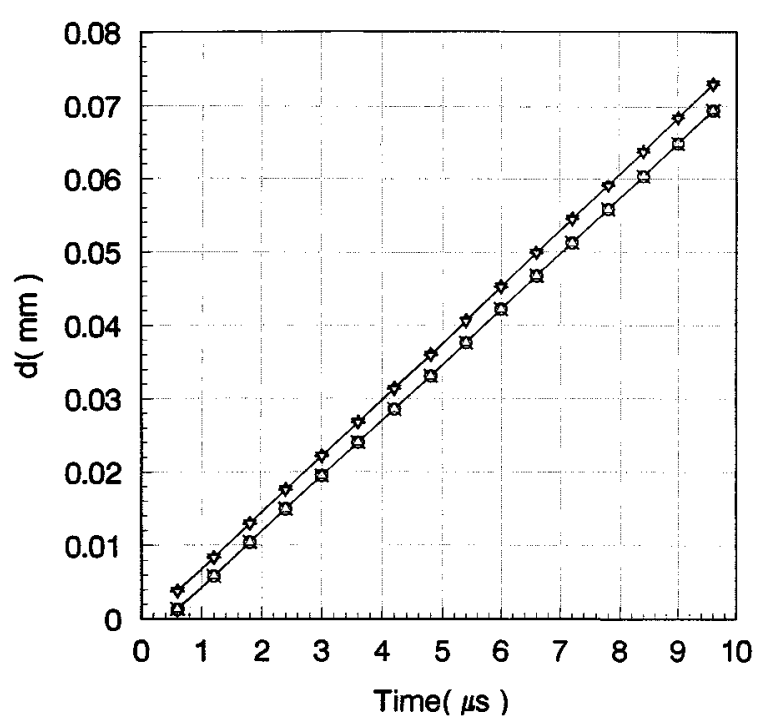

FIG. 5. Position of the plateau versus time. The loading pressure is $26.0 \mathrm{GPa} . \diamond,+$, and $\nabla$ correspond to the forward position of the pressure, velocity, and acoustic speed plateaus, respectively, and $\bigcirc, \times$, and $\triangle$ to the backward position of each.

regimes have the same Chapman-Jouguet (C-J) pressure, approximately $33 \mathrm{GPa}$, and the same detonation velocity, nearly $8220 \mathrm{~m} / \mathrm{s}$, after the shock transfers to detonation. There are two phenomena of interest in Fig. 4. First, as shown in Fig. 4a, the pressure peak may not rise continuously with increasing time in the transition to detonation. Second, there are pressure plateaus as exhibited in Fig. 4d. Figure 5 shows that the variations in both the forward and backward positions of the plateau as a function of time are nearly linear though there are weak differences between the propagation velocities. It is not difficult to describe the governing mechanism for the first phenomenon. When the initial shock is not strong enough, the speed of rarefaction propagating in the explosive charge compacted by the shock wave may be greater than the velocity of the shock front. This results in the rarefaction pursuing and unloading the shock wave. If the rate of heat release is slow, the rarefaction will lead to extinction. How does the plateau set up? Why is it able to be sustained? Figure 4d shows that the rarefaction is not strong enough to completely attenuate the initial shock, which is the essential condition that causes the plateau to form. Figure 6, corresponding to Fig. 4d, shows the profile of acoustic speed, in which $c$ is the relative acoustic speed and $u$ is the velocity of the mass flow. The dashed line is the the detonation velocity versus time in Fig. 6, which has two intersections A and B with each profile of the acoustic speed, as shown in Fig. 7. The point B is the C-J point, and the space between A and B is the width of the reactive zone. Figure 6 shows that the plateau is located behind the reaction region. Its propagation speed is less than the detonation velocity. It is interesting to note that the profiles of the acoustic speed and mass velocity also have the plateau, as shown in Fig. 8. Figure 5 shows that the pressure, mass velocity, and acoustic plateaus are coincident with each other, which means that the propagation speed of the plateaus is identical to the absolute acoustic speed. This is why the plateau is self-sustaining. Do these plateaus result from the effect of the FCT clip or come from a physical mechanism? We are not yet sure. 


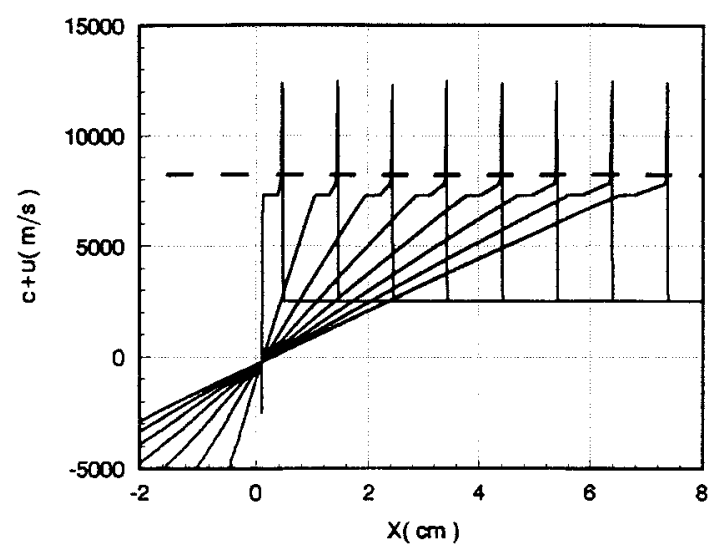

FIG. 6. Shock to detonation transition: acoustic speed profiles. The loading pressure is $26.0 \mathrm{GPa}$.

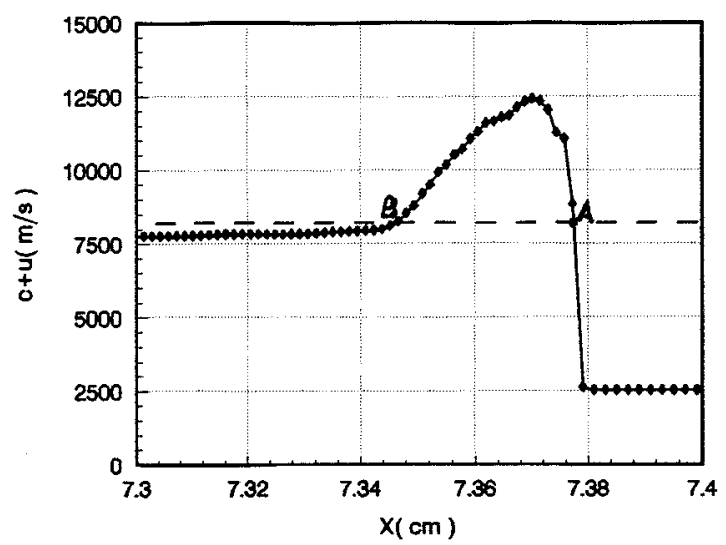

FIG. 7. Shock to detonation transition: acoustic speed profiles near reaction region at $9.0 \mu$ s. The loading pressure is $26.0 \mathrm{GPa}$.

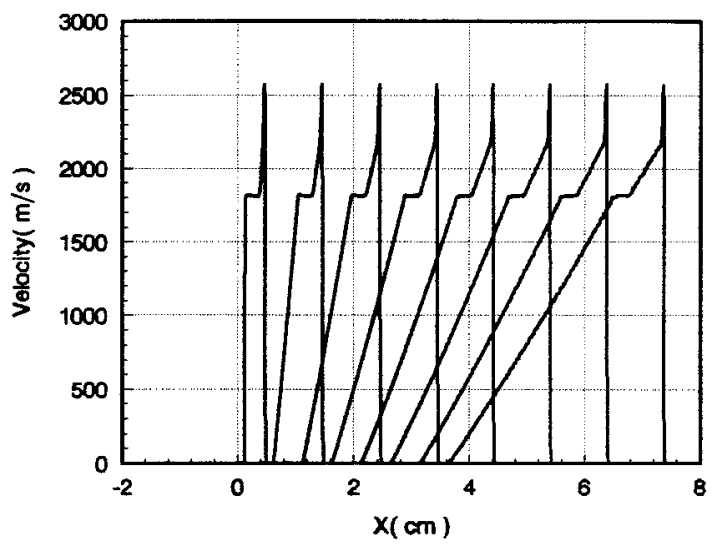

FIG. 8. Shock to detonation transition: mass velocity profiles. The loading pressure is $26.0 \mathrm{GPa}$. 


\section{CONCLUDING REMARKS}

In this work we have formulated an algorithm based on the combination of the Lagrangian finite element discretization with the flux-corrected transport concept. Its accuracy as demonstrated by our numerical experiments makes it a valuable tool for solving the problems of shock dynamics. The forms of the diffusive flux and anti-diffusive flux are very simple because only the nodal velocity is corrected in this algorithm. We found that the simple limiter of Boris and Book $[19,20]$ is effective in correcting overshoots or undershoots that may develop during the time evolution of the solution. In the numerical experiments simulating the shock to detonation transition and detonation propagation, we found that a self-sustaining plateau region may exist in the pressure, velocity, and acoustic profiles. Finally it should be noted that the success of the algorithm is only for the case of one-dimensional shock dynamics. The generalization from 1-D to 2-D and 3-D is the subject of future work.

\section{ACKNOWLEDGMENT}

The authors are grateful for the support from the NNSF of China under Grant K19472011.

\section{REFERENCES}

1. D. S. Stewart, B. W. Asay, and K. Prasad, Simplified modeling of transition to detonation in porous energetic materials, Phys. Fluids 6, 2515 (1994).

2. C. M. Tarver, P. A. Urtiew, and W. C. Tao, Effects of tandem and colliding shock waves on the initiation of triaminotrinitrobenzene, J. Appl. Phys. 78, 3089 (1995).

3. J. A. Zukas, High Velocity Impact Dynamics (Wiley, New York, 1990), p. 609.

4. E. L. Lee and C. M. Tarver, Phenomenological model of shock initiation in heterogeneous explosives, Phys. Fluids 23, 2362 (1980).

5. K. Kim and C. H. Sohn, Modeling of reaction buildup process in shocked porous explosives, in 8th Symposium (International) on Detonation (1985), p. 926.

6. C. M. Tarver, J. O. Hallquist, and L. M. Erickson, Modeling short pulse during initiation of solid explosives, in 8th Symposium (International) on Detonation (1985), p. 951.

7. V. Boyle, R. Frey, and O. Blake, Combined pressure shear ignition of explosives, in 9th Symposium (International) on Detonation (1989), p. 3.

8. C. L. Mader, Recent advances in numerical modeling of detonation, Propell. Explos. Pyrotech. 11, 163 (1986).

9. C. S. Coffey, A prototypical model of a dislocation source capable of generating shear band structures in crystalline solids during shock or impact, J. Appl. Phys. 66, 1654 (1989).

10. D. D. Dlott and M. D. Fayer, Shock molecular solids: Vibrational up pumping, defect hot spots formation, and the onset of chemistry, J. Chem. Phys. 92, 3789 (1990).

11. J. J. Dick, Anomalous shock initiation of detonation in pentaerythritol tetranitrate crystals, J. Appl. Phys. 81, 601 (1991).

12. T. Lappas, A. Leonard, and P. E. Dimotakis, An adaptive Lagrangian method for computing $1 \mathrm{D}$ reacting and non-reacting flows, J. Comput. Phys. 104, 361 (1993).

13. W. Fickett and W. Davis, Detonation (Univ. of California Press, Berkeley, 1979).

14. E. L. Lee and C. M. Tarver, Phenomenological model of shock initiation in heterogeneous explosives, Phys. Fluids 23, 2362 (1980).

15. M. J. Murphy, E. L. Lee, A. M. Weston, and A. E. Williams, Modeling shock initiation in composition B, in 10th International Detonation Symposium, Boston, MA, July 12-16, 1993.

16. P. K. Sweby, Notes on Numerical Fluid Mechanics (Vieweg, Brunswick, 1988), Vol. 24, p. 599. 
17. T. Belytschko and T. J. R. Hughes, Computational methood for transient analysis (North-Holland, Amsterdam, 1983).

18. W. J. Flis, Advanced algorithms for computer simulation of hypervelocity impact, J. Impact Engrg. 5, 269 (1987).

19. J. P. Boris and D. L. Book, Flux-corrected transport. I. SHASTA, a transport algorithm that works, J. Comput. Phys. 11, 38 (1973).

20. D. L. Book, J. P. Boris, and K. Hain, Flux-corrected transport. II. Generalizations of the method, J. Comput. Phys. 18, 248 (1975).

21. S. T. Zalesak, Fully multidimensional flux-corrected transport algorithm for fluids, J. Comput. Phys. 31, 335 (1979).

22. B. E. McDonald, Pseudospectral method for scalar hyperbolic conservation laws, J. Comput. Phys. 82, 413 (1989).

23. J. Giannakouros and G. E. Karniadakis, A spectral element-FCT method for the compressible Euler equation, J. Comput. Phys. 115, 65 (1994).

24. D. Odstrčil, Improved FCT algorithm for shock hydrodynamics, J. Comput. Phys. 115, 65 (1994).

25. K. Salari and S. Steinberg, Flux-corrected transport in a moving grid, J. Comput. Phys. 111, 24 (1994).

26. R. Löhner, K. Morgan, J. Peraire, and M. Vahdati, Finite element flux-corrected transport (FEM-FCT) for the Euler and Navier-Stokes equations, Int. J. Numer. Methods Fluids 7, 1093 (1987).

27. J. J. Ambrosiano, S. T. Brandon, R. Löhner, and C. R. DeVore, Electromagnetics via the Taylor-Galerkin finite element method on unstructured grids, J. Comput. Phys. 110, 310 (1994). 